\title{
Avaliação de conhecimentos sobre exposição solar
}

Rita Meireles Pedro, ${ }^{1}$ Catarina Sá Couto, ${ }^{2}$ Daniela Almeida Ribeiro, ${ }^{3}$ Mafalda Oliveira, ${ }^{4}$ Raquel Lisboa, ${ }^{5}$ Sandra Mimoso Guedes ${ }^{6}$

\section{RESUMO}

Objetivos: Caracterizar o conhecimento dos utentes sobre os cuidados associados à exposição solar, os fatores de risco e sinais de alarme para neoplasia cutânea. Estudar a associação entre as variáveis: idade, sexo, escolaridade, fotótipo, antecedentes de neoplasia cutânea (pessoais ou em contactos próximos) e o nível de conhecimento dos utentes.

Métodos: Estudo transversal. Durante os meses de abril e maio de 2018 foram entregues questionários de autopreenchimento, compostos por dados sociodemográficos, questões teóricas sobre exposição solar e seus riscos, fatores de risco e sinais de alarme para neoplasia cutânea aos utentes com idade igual ou superior a 18 anos que compareceram à consulta programada de seis Unidades de Saúde Familiar (USF). Foi realizada a análise estatística das suas variáveis, utilizando o software estatístico IBM SPSS Statistics ${ }^{\circledR}$, assumindo-se um nível de significância de $5 \%$.

Resultados: Participaram 435 indivíduos, dos quais 29 não completaram o questionário, resultando numa amostra final de 406 indivíduos. Os inquiridos apresentaram uma média de idades de 47士17,1 anos (mínimo: 18; máximo: 89 anos) e 67\% eram do sexo feminino. A pontuação média no questionário foi de $9,3 \pm 2,06$ pontos (sendo a pontuação máxima de 13 valores), mais elevada nos inquiridos com idades entre 35 e 55 anos, nos mais escolarizados e nos inquiridos com fototipo 3 . Os inquiridos mais velhos e aqueles com menor nível de escolaridade associaram-se a uma pontuação baixa a intermédia no questionário $(p<0,001)$. Os inquiridos com conhecidos com diagnóstico de cancro de pele e os inquiridos com pele mais clara obtiveram uma pontuação mais elevada. No entanto, após ajuste para potenciais confundidores, verificou-se que apenas as variáveis escolaridade e tipo de pele mantiveram associação com o nível de conhecimentos.

Conclusões: O nível de conhecimentos sobre a exposição solar encontrado é satisfatório, ainda que existam lacunas importantes relativamente ao conhecimento de formas de proteção, de fatores que aumentam o risco de cancro cutâneo e de sinais de alarme. Estes resultados corroboram a pertinência do estudo, evidenciando a relevância da realização de intervenções de educação para a saúde sobre o tema, considerando o seu previsível impacto na incidência e prevalência de neoplasia cutânea. Salienta-se ainda, na sequência deste estudo, a pertinência da realização de uma investigação sobre comportamentos da população relativamente à exposição e proteção solar.

Palavras-chave: Neoplasia cutânea; Prevenção; Questionário; Fatores de risco.

\section{INTRODUÇÃO}

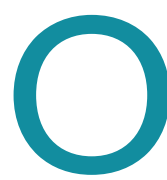

cancro da pele é o tipo de cancro mais frequente nos indivíduos com fototipos mais claros e o mais comum no mundo, com incidência crescente. ${ }^{1-2}$ É também um dos tipos de cancro mais facilmente evitável, uma vez que o seu principal fator de risco, a exposição à radiação ultravioleta (UV), pode ser reduzido com alterações de compor-

1. USF Horizonte, ULS Matosinhos.

2. USF Porta do Sol, ULS Matosinhos.

3. USF Leça, ULS Matosinhos.

4. USF Santo André de Canidelo, ACeS Grande Porto VII/Gaia.

5. USF Monte Crasto, ACeS Gondomar.

6. USF Ramalde, ACeS Porto Ocidental. tamento em relação à exposição solar. ${ }^{3-4}$ Quando tratado nas fases iniciais apresenta elevadas taxas de cura, pelo que se torna crucial um diagnóstico precoce..$^{1-2}$

A consciência pública sobre os cuidados a ter com a pele e os sintomas do cancro cutâneo é ainda reduzida. ${ }^{3}$ Na verdade, tem aumentado a incidência de queimaduras solares e a prática de bronzeamento artificial. ${ }^{5}$ A valorização estética do bronzeado, por vezes associada a mensagens veiculadas pelos meios de comunicação social, pode levar à exposição solar prolongada e, por vezes, desprotegida. ${ }^{5}$

Portugal é um dos países com maior exposição solar da Europa, pelo que não deve ser esquecido o maior risco potencial nos funcionários que trabalham no 
exterior (como agricultores, polícias, etc.) devido a uma maior exposição à radiação $U V^{6}$

Estudos realizados concluíram não existir evidência suficiente que aconselhe o clínico a observar a pele como forma de rastreio do cancro da pele, dando ênfase à importância do autoexame. ${ }^{7}$ No entanto, parece existir um impacto positivo com as intervenções educacionais na prevenção do cancro da pele. ${ }^{8-9}$

Foram já realizados alguns estudos noutras populações para avaliar o nível de conhecimentos nesta área. Alguns dos resultados apresentados foram contraditórios, mas em vários estudos concluiu-se que o conhecimento da população não era o mais adequado. ${ }^{10-13}$

De acordo com estes dados, considera-se importante avaliar o nível de conhecimento dos utentes em relação à prevenção do cancro cutâneo e sinais/sintomas deste tipo de neoplasia, de forma a caracterizar a necessidade de realizar intervenções educacionais na população.

\section{MÉTODOS}

Foi conduzido um estudo transversal numa população com idade igual ou superior a 18 anos, pertencente a seis unidades de saúde do Grande Porto, no Norte de Portugal, envolvendo um total de 75.008 pessoas.

A técnica de amostragem utilizada foi probabilística, com seleção de uma amostra aleatória sistemática. Estabeleceu-se como critérios de inclusão: utentes com idade igual ou superior a 18 anos que comparecessem à consulta programada da sua USF no período de abril e maio de 2018. Foram considerados critérios de exclusão: ser analfabeto, apresentar a linguagem, cognição ou órgãos sensoriais afetados e/ou não falar português.

O tamanho amostral foi determinado considerando um intervalo de confiança de $95 \%$ e um nível de precisão de $5 \%$, perfazendo um total de 383 indivíduos. Tendo em conta os possíveis omissos, foi incrementado $10 \%$ do tamanho amostral, perfazendo no total uma amostra final de 421 indivíduos.

Foi aplicado um questionário de autopreenchimento em português, elaborado pelas autoras, com base na revisão bibliográfica efetuada e na opinião de um perito em dermatologia, o qual foi submetido previamente a um teste piloto. Este teste foi aplicado a um grupo de indivíduos de dimensão correspondente a cerca de $10 \%$ da amostra calculada (40 indivíduos), com características semelhantes às da população em estudo. No questioná- rio foram incluídos dados sociodemográficos e questões teóricas sobre exposição solar e seus riscos, bem como fatores de risco e sinais de alarme para neoplasia cutânea. As variáveis estudadas foram: idade, sexo, estado civil, escolaridade, profissão, antecedentes pessoais e/ou de contactos próximos de cancro da pele, fototipo, exposição solar e envelhecimento cutâneo, risco de queimadura solar com o nevoeiro, roupa opaca e protetor solar, tamanho da sombra e segurança de exposição solar, horário de exposição solar, protetor solar e estação do ano, quando aplicar protetor solar, frequência de aplicação de protetor solar na praia e necessidade de aplicação quando sob guarda-sol, necessidade de cuidados de proteção solar em indivíduos com fototipos mais elevados, formas de proteção solar, fatores de risco de neoplasia cutânea e sinais de alarme num nevo.

Procedeu-se à entrega do questionário aos utentes elegíveis no final da consulta médica, de numeração ordinal ímpar no agendamento de cada médico das várias USF (USF Horizonte, USF Leça e USF Porta do Sol - ULSM; USF Monte Crasto - ACeS de Gondomar; USF Ramalde - ACeS Porto Ocidental; USF Santo André de Canidelo-ACeS Grande Porto VII/Gaia). Os utentes foram informados quanto à realização do estudo e esclarecidos relativamente à conduta a adotar para a devolução do questionário, após o seu preenchimento (colocação em caixa própria e identificada para o efeito, na secretaria de cada USF). Todos os procedimentos foram realizados considerando os princípios éticos, tendo este trabalho sido autorizado pelas Comissões de Ética da ULS Matosinhos e da ARS Norte.

Os dados foram submetidos a uma análise descritiva, que incluiu o cálculo de frequências e percentagens, bem como de medidas de tendência central e de dispersão. Os fototipos cutâneos foram avaliados através da Classificação de Fitzpatrick e, posteriormente, recodificados em duas categorias, dada a baixa prevalência de inquiridos com o fototipo 1, 5 ou 6. Assim, obtiveram-se as categorias "pele clara» (incluindo os fototipos 1, 2 e 3) e «pele morena, escura ou negra» (incluindo os fototipos 4,5 e 6). A avaliação global dos conhecimentos dos inquiridos teve em consideração 13 questões de escolha múltipla (resposta única ou múltipla). As respostas foram dicotomizadas em corretas e incorretas, com atribuição de 1 valor a cada questão respondida corretamente. No caso das questões de 


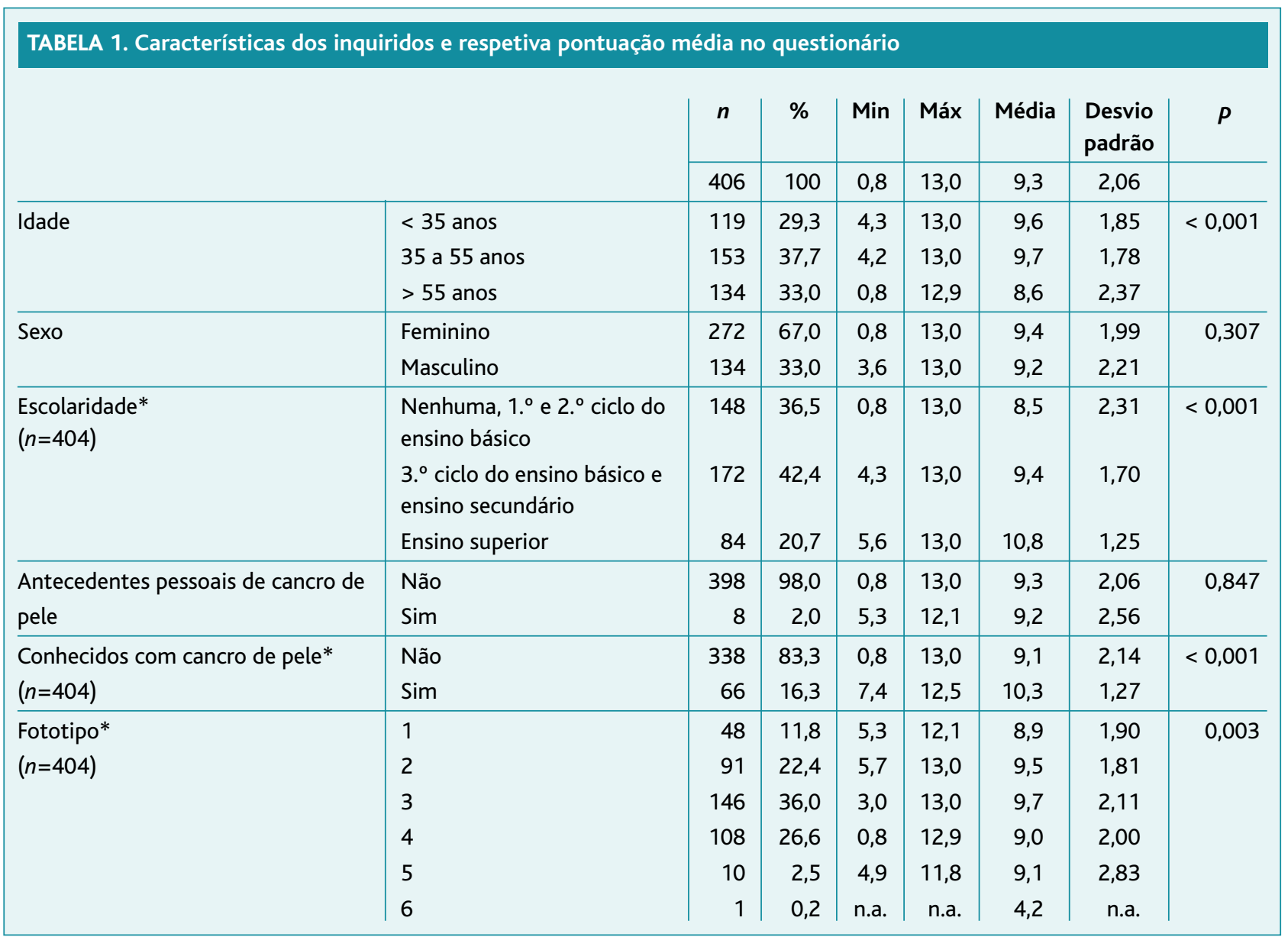

Legenda: *Nesta variável, a soma do número de inquiridos não soma 406 devido à existência de valores omissos; n.a. = não aplicável.

resposta múltipla, o valor de cada questão foi dividido pelo número de hipóteses corretas, de modo a obter uma cotação para cada resposta certa assinalada. Posteriormente foram somados os pontos de todas as questões, obtendo-se assim uma pontuação total dos conhecimentos (para um máximo possível de 13 valores). O nível de conhecimentos foi categorizado em três níveis, consoante a pontuação obtida: baixo ( $<5$ pontos), intermédio ( 5 a 10 pontos) e elevado ( $\geq 10$ pontos). A pontuação média obtida no questionário foi calculada para diferentes grupos de indivíduos no que se refere à idade, ao sexo, ao nível de escolaridade, aos antecedentes pessoais, à existência de conhecidos com cancro de pele e ao tipo de pele. A divisão das respostas a cada pergunta pelo nível de escolaridade baseou-se no facto de esta constituir um indicador de literacia. ${ }^{14} \mathrm{Re}-$ correu-se ao teste $t$ de Student para amostras independentes e à análise de variância (ANOVA) para comparar a média entre dois ou três grupos, respetivamente. Utilizou-se a regressão logística binária para estimar a associação entre as características e a pontuação baixa a intermédia no questionário, através de Odds Ratio (OR) e respetivos intervalos de confiança a $95 \%$ (IC95\%). Foi utilizado o software estatístico IBM SPSS Statistics ${ }^{\circledR}$, v. 24.0, assumindo-se um nível de significância de $5 \%$.

\section{RESULTADOS}

No presente estudo participaram 435 indivíduos, dos quais 29 não completaram o questionário, resultando numa amostra final de 406 indivíduos.

Os inquiridos apresentaram uma média de idades de 
47ะ17,1 anos (mínimo: 18; máximo: 89 anos) e 67,0\% eram do sexo feminino. Cerca de $36,5 \%$ frequentou a escola até ao final do segundo ciclo do ensino básico e $20,7 \%$ dos inquiridos completou um grau académico. A maioria dos inquiridos nunca teve cancro de pele $(98,0 \%)$ e não tinha conhecidos com esse diagnóstico $(83,3 \%)$. Relativamente aos fototipos cutâneos (Classificação de Fitzpatrick) entre os inquiridos, o mais comum foi o $3(36,0 \%)$, seguido do $4(26,6 \%)$ e do 2 $(22,4 \%)$. Apenas um inquirido apresentou fototipo 1.

A pontuação média no questionário de conhecimentos sobre os cuidados e riscos associados à expo-

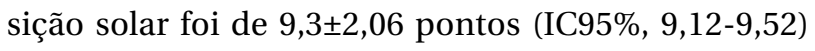
(sendo a pontuação máxima de 13 valores), mais elevada nos inquiridos com idades entre 35 e 55 anos, nos mais escolarizados e nos inquiridos com fototipo 3 ( Tabela I). Na categoria de pontuação mais baixa $(<5$ pontos) incluem-se 3,9\% dos inquiridos, sendo que 57,4\% se enquadra na categoria intermédia (5 a 10 pontos) e $38,7 \%$ na mais elevada ( $\geq 10$ pontos).

Os inquiridos mais velhos e os que apresentam menor nível de escolaridade associaram-se a uma pontuação baixa a intermédia no questionário $(p<0,001)$. Por outro lado, os inquiridos com conhecidos com diagnóstico de cancro de pele e aqueles com pele mais clara obtiveram uma pontuação mais elevada ( $p<0,001 \mathrm{e}$ $p=0,003$, respetivamente). No entanto, após ajuste para idade, escolaridade, conhecidos com cancro de pele e tipo de pele, verificou-se que apenas mantiveram a associação o nível de escolaridade (Nenhuma, $1 .^{\circ} \mathrm{e} 2 .^{\circ} \mathrm{ci}-$ clo do ensino básico $v s$. Ensino superior - $\mathrm{OR}=10,75$ [IC95\%, 5,34-21,64]; $3 .^{\circ}$ ciclo do ensino básico e ensino secundário vs. Ensino superior - OR=6,54 [IC95\%, 3,54$12,09]$ ) e o tipo de pele (pele morena, escura ou negra vs. pele clara - OR=1,94 [IC95\%, 1,15-3,25]) - Tabela II.

As Figuras 2 a 5 apresentam a percentagem de respostas corretas a cada pergunta do questionário (cuidados de exposição solar, fatores de risco e sinais de alarme para neoplasia cutânea), divididas pelo nível de escolaridade. Globalmente, observa-se uma proporção mais elevada de respostas corretas à medida que a escolaridade aumenta. De uma forma geral, os indivíduos não reconhecem a roupa opaca como uma barreira à exposição solar, mesmo os mais escolarizados (destes, apenas $41,7 \%$ respondeu corretamente). Nos indivíduos menos escolarizados, a sombra mais pequena não é associada a um perigo por
57,4\% dos inquiridos e a frequência de aplicação do protetor solar na praia, bem como a sua utilização não apenas no Verão, são subvalorizadas (apenas reconhecidos por $59,5 \%$ e $61,5 \%$, respetivamente).

Entre o chapéu, o protetor solar e os óculos de sol, estes últimos são os menos reconhecidos como protetores da exposição solar, particularmente entre os menos escolarizados (32,4\% não identifica).

No que diz respeito à identificação dos potenciadores do risco de cancro de pele, apenas 28 dos 406 inquiridos identificaram corretamente todas as opções, sendo a opção menos pontuada a referente à idade superior a 50 anos (77,3\% dos inquiridos não a assinalou). As cicatrizes ou queimaduras na pele e a exposição solar intensa durante um curto período de tempo são pouco reconhecidas como constituindo um risco (particularmente no grupo menos escolarizado, no qual ambos apenas são identificados por cerca de um terço dos inquiridos).

Relativamente às características dos sinais que devem levar a consultar o médico, apenas 38 inquiridos identificaram todas as opções, sendo a característica menos identificada a textura áspera (69,5\% não a assinalou). Destacam-se o crescimento rápido e o facto de se assemelhar a uma ferida, mas não cicatrizar, como sendo as características mais associadas a neoplasia cutânea.

Note-se que os inquiridos mais escolarizados assinalaram mais opções do que os indivíduos menos escolarizados (em ambas as questões de oito respostas, referentes aos fatores de risco e sinais de alarme para neoplasia cutânea, em média os mais escolarizados assinalaram corretamente cinco respostas, enquanto os menos escolarizados apenas três).

\section{DISCUSSÃO}

Grande parte dos inquiridos demonstrou um nível de conhecimentos satisfatório sobre os cuidados e riscos da exposição solar, sendo este nível mais elevado nos inquiridos com idades entre 35 e 55 anos, nos mais escolarizados e nos inquiridos com fototipo 3, ainda que existam lacunas importantes registadas ao nível do conhecimento de formas de proteção e de fatores que aumentam o risco de desenvolvimento de cancro de pele. Ao nível dos sinais de alarme para neoplasia cutânea, o conhecimento demonstrado foi escasso.

Estes resultados foram semelhantes aos obtidos por Hora no seu estudo realizado no Brasil, ${ }^{12}$ no qual se 


\begin{tabular}{|c|c|c|c|}
\hline & & $\begin{array}{l}\text { OR bruto } \\
\text { (IC95\%) }\end{array}$ & $\begin{array}{l}\text { OR ajustado } \\
\text { (IC95\%) }\end{array}$ \\
\hline Idade & $\begin{array}{l}<35 \text { anos } \\
35 \text { a } 55 \text { anos } \\
>55 \text { anos }\end{array}$ & $\begin{array}{c}\text { Ref. } \\
1,07(0,66-1,74) \\
1,96(1,60-3,31)\end{array}$ & $\begin{array}{c}\text { Ref. } \\
0,93(0,53-1,63) \\
1,20(0,63-2,30)\end{array}$ \\
\hline Sexo & $\begin{array}{l}\text { Feminino } \\
\text { Masculino }\end{array}$ & $\begin{array}{c}\text { Ref. } \\
0,92(0,60-1,40)\end{array}$ & $\begin{array}{c}\text { Ref. } \\
0,85(0,53-1,37)\end{array}$ \\
\hline Escolaridade & $\begin{array}{l}\text { Ensino superior } \\
3 .^{\circ} \text { ciclo do ensino básico e ensino secundário } \\
\text { Nenhuma, } 1 .^{\circ} \text { e } 2 .^{\circ} \text { ciclo do ensino básico }\end{array}$ & $\begin{array}{c}\text { Ref. } \\
6,61(3,62-12,06) \\
12,19(6,40-23,21)\end{array}$ & $\begin{array}{c}\text { Ref. } \\
6,54(3,54-12,09) \\
10,75(5,34-21,64)\end{array}$ \\
\hline $\begin{array}{l}\text { Antecedentes pessoais de cancro } \\
\text { de pele }\end{array}$ & $\begin{array}{l}\text { Não } \\
\text { Sim }\end{array}$ & $\begin{array}{c}\text { Ref. } \\
0,63(0,15-2,54)\end{array}$ & $\begin{array}{c}\text { Ref. } \\
0,93(0,18-4,93)\end{array}$ \\
\hline Conhecidos com cancro de pele & $\begin{array}{l}\text { Não } \\
\text { Sim }\end{array}$ & $\begin{array}{c}\text { Ref. } \\
0,46(0,27-0,78)\end{array}$ & $\begin{array}{c}\text { Ref. } \\
0,60(0,33-1,11)\end{array}$ \\
\hline Tipo de pele & $\begin{array}{l}\text { Clara } \\
\text { Morena, escura ou negra }\end{array}$ & $\begin{array}{c}\text { Ref. } \\
1,82(1,15-2,90)\end{array}$ & $\begin{array}{c}\text { Ref. } \\
1,94(1,15-3,25)\end{array}$ \\
\hline
\end{tabular}

Legenda: *Ajustado para: idade, escolaridade, conhecidos com cancro de pele e tipo de pele (todas categóricas, de acordo com a tabela); Ref. = categoria de referência.

verificou que $87,3 \%$ dos entrevistados se diziam conhecedores dos efeitos prejudiciais da exposição solar e esta percentagem aumentava com o nível de escolaridade. Esta associação também foi observada por Seité no seu inquérito internacional, ${ }^{13}$ sendo que as mulheres pareceram melhor informadas, o que não se observou no presente estudo. Nesteinquérito, os sinais precoces de cancro cutâneo também não eram universalmente conhecidos.

A principal limitação deste trabalho é ter como instrumento de medida um questionário não validado na sua globalidade. Assim, algumas questões poderão eventualmente não refletir a interpretação e tradução exata do conhecimento que se pretendia avaliar, dificultando a generalização dos resultados para além da amostra estudada, apesar de ter sido precedida de uma validação das questões em prova piloto e consultados peritos na área da dermatologia. Também a caracterização dos inquiridos quanto ao fototipo cutâneo é digna de nota, na medida em que a autoavaliação da cor de pele e a identificação do respetivo fototipo são dados subjetivos e controversos, pelo que deveriam ideal- mente ser avaliados pelas investigadoras.

De acordo com o já demonstrado em estudos anteriores, ${ }^{12-13}$ a perceção e o conhecimento sobre os riscos da exposição solar excessiva, por si só, não parece ser suficiente para garantir hábitos de vida e comportamentos adequados de proteção solar. Este estudo é pertinente, na medida em que analisa a literacia da população sobre um problema de saúde prevalente e poderá servir de base para projetos de intervenção para correção de comportamentos de risco, assim como para um estudo posterior comparativo entre os conhecimentos e os comportamentos da população relativos à exposição e proteção solar.

Atualmente não existem recomendações para o rastreio sistemático do cancro de pele realizado pelo médico em adultos, dado que a evidência é limitada relativamente à redução da mortalidade e existe dano potencial que não é desprezível. ${ }^{7}$ Recomenda-se, portanto, o enfoque no aconselhamento pessoal por parte do médico aos adultos jovens, principalmente àqueles que tenham a pele clara, sobre como minimizar a sua ex- 


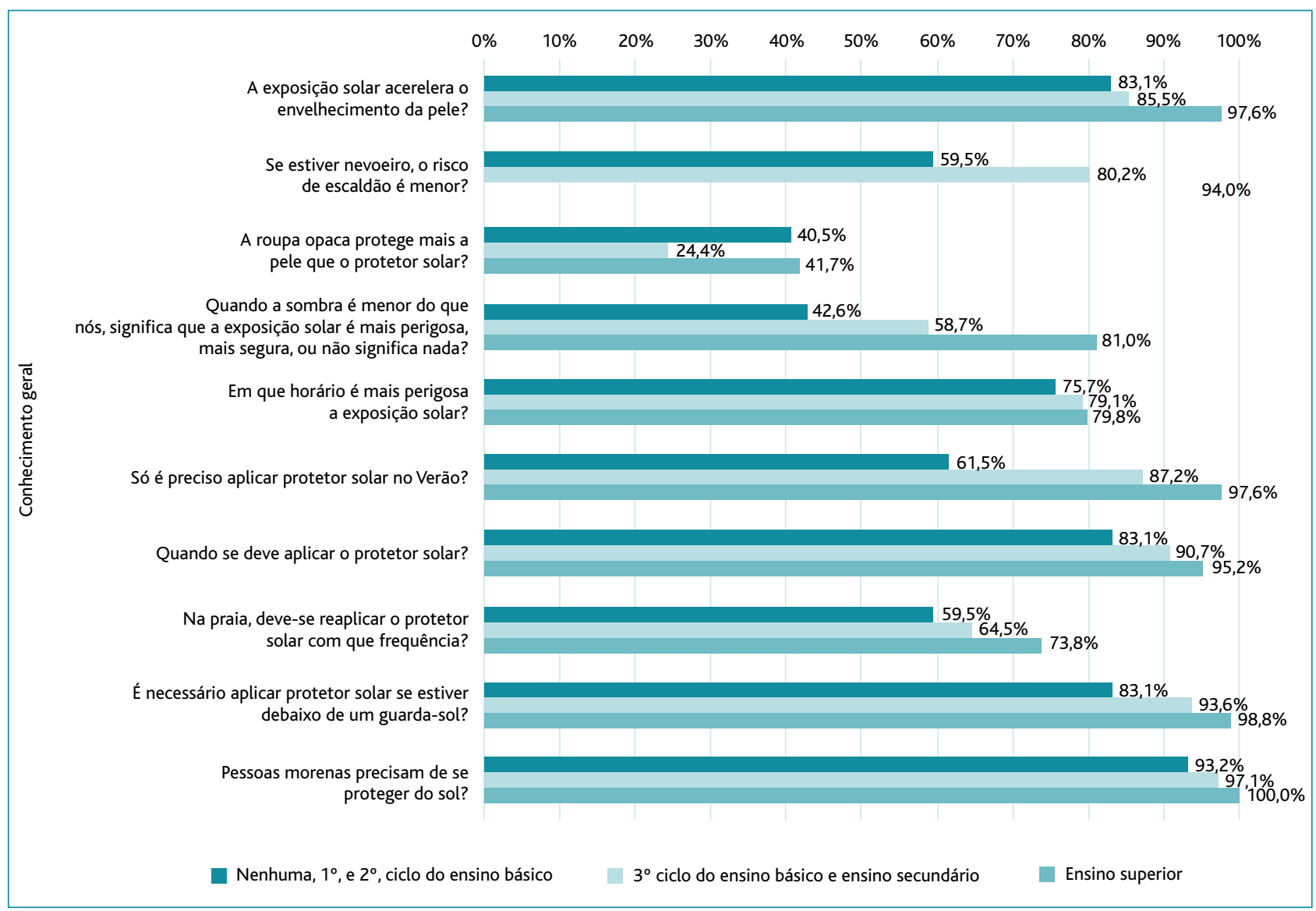

Figura 1. Percentagem de respostas corretas a cada pergunta de conhecimento geral, de acordo com o nível de escolaridade dos inquiridos.

posição solar, de forma a reduzir o risco de cancro. ${ }^{15}$

A população estudada neste artigo demonstrou algumas falhas importantes que devem ser colmatadas por parte dos clínicos em consulta, de forma individual e sistemática, dando enfoque a populações mais jovens e com mais fatores de risco.

\section{REFERÊNCIAS BIBLIOGRÁFICAS}

1. Sociedade Portuguesa de Dermatologia e Venereologia. Cancros da pele e dermatoses pré-cancerosas [homepage]. Lisboa: SPDV; [cited 2017 Apr]. Available from: https://www.spdv.pt/_doencas_de_pele_2

2. Leiter U, Eigentler T, Garbe C. Epidemiology of skin cancer. In: Reichrath J, editor. Sunlight, vitamin D and skin cancer. 2nd ed. New York: Springer; 2014. p. 120-40. ISBN 9781493904365

3. Euromelanoma. Learn about skin cancer [homepage]. Brussels: Euromelanoma; [cited 2017 Apr]. Available from: https://www.euromelanoma.org/intl/node/8

4. Silva HP, Ferrão C. Cancro da pele: podemos preveni-lo? [Skin cancer: can we prevent it?]. Rev Port Clin Geral. 2003;19(5):485-90. Portuguese
5. Neves AC, Ferreira GM, Teixeira MC, Monteiro E. Detecção precoce de neoplasias cutâneas em cuidados de saúde primários: relato de uma experiência [Early detection of skin cancer in primary care: an experience]. Rev Port Clin Geral. 2011;27(4):381-7. Portuguese

6. Apalla Z, Lallas A, Sotiriou E, Lazaridou E, loannides D. Epidemiological trends in skin cancer. Dermatol Pract Concept. 2017;7(2):1-6.

7. Bibbins-Domingo K, Grossman DC, Curry SJ, Davidson KW, Ebell M, Epling JW Jr, et al. Screening for skin cancer: US Preventive Services Task Force Recommendation Statement. JAMA. 2016;316(4):429-35.

8. Falk $M$, Anderson C. Prevention of skin cancer in primary healthcare: an evaluation of three different prevention effort levels and the applicability of a phototest. Eur J Gen Pract. 2008;14(2):68-75.

9. Lin JS, Eder M, Weinmann S. Behavioral counseling to prevent skin cancer: a systematic review for the U.S. Preventive Services Task Force. Ann Intern Med. 2011;154(3):190-201.

10. Mourão P. Melanoma maligno: estudo comparativo entre o nível de conhecimentos e comportamentos preventivos nos alunos do $9^{\circ}$ ano da área do Centro de Saúde de São João [Malignant melanoma: knowledge and behavior of 9th grade students in the S. Joao Health Centre]. Rev Port Clin Geral. 2002;18(4):203-16. Portuguese 
11. Castilho IG, Sousa MA, Leite RM. Fotoexposição e fatores de risco para câncer da pele: uma avaliação de hábitos e conhecimentos entre estudantes universitários [Photoexposure and risk factors for skin cancer: an evaluation of behaviors and knowledge among university students]. An Bras Dermatol. 2010;85(2):173-8. Portuguese

12. Hora C, Batista CV, Guimarães PB, Siqueira R, Martins S. Avaliação do conhecimento quanto a prevenção do câncer da pele e sua relação com exposição solar em frequentadores de academia de ginástica, em Recife [Evaluation about knowledge of skin cancer prevention and its relation with sun exposure among gym members in the city of Recife]. An Bras Dermatol. 2003;78(6):693-701. Portuguese

13. Seité S, Del Marmol V, Moyal D, Friedman AJ. Public primary and secondary skin cancer prevention, perceptions and knowledge: an international cross-sectional survey. J Eur Acad Dermatol Venereol. 2017;31(5):815-20.

14. Pedro AR, Amaral $O$, Escoval $A$. Literacia em saúde, dos dados à ação: tradução, validação e aplicação do European Health Literacy Survey em Portugal [Health Literacy, from data to action: translation, validation and application of the European Health Literacy Survey in Portugal]. Rev Port Saúde Pública. 2016;34(3):259-75. Portuguese

15. Moyer VA, US Preventive Services Task Force. Behavioral counseling to prevent skin cancer: US Preventive Services Task Force recommendation statement. Ann Intern Med. 2012;157(1):59-65.

\section{CONFLITO DE INTERESSES}

Os autores declaram não ter quaisquer conflitos de interesse.

\section{ENDEREÇO PARA CORRESPONDÊNCIA}

Rita Meireles Pedro

E-mail: rita_meireles90@hotmail.com

https://orcid.org/0000-0003-0984-3429

Recebido em 13-03-2019

Aceite para publicação em 10-01-2020

\section{ABSTRACT}

\section{ASSESSMENT OF KNOWLEDGE ABOUT SOLAR EXPOSURE}

Objectives: To characterize the patient's knowledge about the care associated with sun exposure, risk factors, and alarm signs for cutaneous neoplasia. To study the association between the age, sex, education, phototype, and history of cutaneous neoplasia (personal or in close contacts) and the level of the patient's knowledge.

Methods: Cross-sectional study. From April to May of 2018, self-administered questionnaires, composed of sociodemographic data, theoretical questions about sun exposure and its risks, risk factors, and alarm signals for cutaneous neoplasia, were delivered to patients aged 18 years and over who attended the scheduled consultation in six Family Health Units. A statistical analysis of the variables was performed. It was used the statistical software IBM SPSS Statistics ${ }^{\circledR}$, assuming a level of significance of $5 \%$.

Results: A total of 435 individuals participated, of whom 29 did not complete the questionnaire, resulting in a final sample of 406 individuals. Participants had a mean age of $47 \pm 17.1$ years (minimum: 18 , maximum: 89 years) and $67 \%$ were female. The mean score on the questionnaire was $9.3 \pm 2.06$ points (with the maximum score being 13 points). The highest scores corresponded to participants with 35-55 years of age, a higher level of education, and phototype 3. The older participants and those with lower levels of education were associated with a low to intermediate score on the questionnaire. Participants that have contacts with a skin cancer diagnosis and those with lighter skin scored higher. However, after adjusting for potential confounders, only the level of education and the skin type maintained the association.

Conclusions: There is a satisfactory level of knowledge about sun exposure, although there are important gaps regarding the knowledge of forms of protection, factors that increase the risk of skin cancer and alarm signs. These results corroborate the pertinence of the study, evidencing the relevance for the implementation of health education interventions on the topic, considering its predicted impact on the incidence and prevalence of cutaneous neoplasia. Moving forward, the relevance of conducting research on population behaviors regarding exposure and sun protection is also highlighted.

Keywords: Cutaneous neoplasia; Prevention; Questionnaire; Risk factors. 


\section{ANEXO I \\ Questionário}

Está a decorrer na USF um estudo de investigação sobre os conhecimentos das pessoas sobre os cuidados e riscos associados à exposição solar. Pretendemos contribuir para uma melhoria dos conhecimentos da população sobre este assunto, e é por isso que a sua colaboração é fundamental.

Assim, convidamo-lo(a) a participar como voluntário(a) neste estudo, respondendo a um questionário. O seu preenchimento demora cerca de 5 minutos.

Pode preenchê-lo enquanto espera por ser atendido na USF,

- Idade: anos

- Sexo:

Feminino

Masculino

- Estado Civil:

Solteiro

Casado/União de facto

Separado/divorciado

Viúvo

- Escolaridade:

- Profissão

- Já teve ou tem cancro da pele?
$\square$ Sim
Não

- Alguém seu conhecido tem ou já teve cancro da pele?
Sim
Não

- Qual o seu tipo de pele? (escolha a opção que mais se adequa)

- A exposição solar acelera o envelhecimento da pele?

- Se estiver nevoeiro, o risco de escaldão é menor:

- Roupa opaca protege mais a pele que o protetor solar? em casa, ou noutro local onde lhe seja mais conveniente. Depois de preenchido, hoje ou noutro dia, agradecíamos que o colocasse na caixa fechada e devidamente identificada, colocada ao lado do secretariado.

Os dados recolhidos serão usados exclusivamente para a presente investigação, sendo que a confidencialidade e o anonimato serão sempre assegurados.

Agradecemos a sua colaboração. Poderá esclarecer qualquer dúvida junto do seu Médico ou Enfermeiro de Família.

- Quando a sombra é menor do que nós, significa que a exposição solar é:

- Em que horário é mais perigosa a exposição solar?

- Só é preciso aplicar protetor solar no Verão?

- Quando se deve aplicar o protetor solar? (escolha a opção mais adequada)

- Na praia deve-se reaplicar o protetor solar com que frequência?

- É necessário aplicar protetor solar se estiver debaixo de um guarda-sol?

- Pessoas morenas precisam de se proteger do sol?

- O que é importante usar quando se expõe ao sol? (pode escolher mais do que uma)

- Selecione as opções que podem aumentar o risco de cancro da pele (pode escolher mais do que uma):

- Selecione as características de um sinal na pele que considera que devam levar a consultar o seu médico (pode escolher mais do que uma): 